\title{
Influence of temperature and crystal orientation on tool wear during single point diamond turning of silicon
}

\author{
Saurav Goel, Xichun Luo*, Robert L. Reuben, Hongmin Pen \\ School of Engineering and Physical Sciences, Heriot-Watt University, Edinburgh EH144AS, Scotland, UK
}

\section{A R T I C L E I N F O}

\section{Article history:}

Received 13 June 2011

Received in revised form 10 February 2012

Accepted 13 February 2012

Available online 21 February 2012

\section{Keywords:}

MD simulation

Single point diamond turning

Single crystal silicon

Tool wear

Crystal orientation

\begin{abstract}
A B S T R A C T
Owing to the capricious wear of cutting tools, ultra precision manufacturing of silicon through single point diamond turning (SPDT) operation becomes a challenging task. It thus becomes non-trivial to understand the contribution of temperature and crystal orientation during the SPDT process in order to suppress tool wear. Molecular dynamics (MD) simulation is an appropriate tool to study nanoscale processes occurring at the femtosecond/picosecond timescale which cannot otherwise be studied experimentally or by the finite element method (FEM). Accordingly, MD simulation has been deployed with a realistic analytical bond order potential (ABOP) formalism based potential energy function to simulate the single point diamond turning operation of single crystal silicon in order to understand the influence of temperature and crystal orientation on the tool wear mechanism. Results showed the strong influence of crystal orientation on the wear resistance of a diamond tool; cubic orientation performed better than dodecahedral orientation. It was also observed that high pressure phase transformation (HPPT) in the cutting zone was accompanied by the formation of dangling bonds of silicon. Under the influence of cutting temperature, the newly formed dangling bonds of silicon chemically combine with the pre-existing dangling bonds on the surface of the diamond tool resulting in the formation of silicon carbide ( $\mathrm{SiC}$ ), the main appearance of which was evident at the tool flank face. Continuous abrasion of the diamond cutting tool with SiC causes $s p^{3}-s p^{2}$ disorder of the diamond tool. Hence, both these processes proceed in tandem with each other. The mechanism proposed here is in good agreement with a recent experimental study, where silicon carbide and carbon like particles were observed using X-ray photoelectron spectroscope (XPS) technology after machining a silicon wafer with a diamond tool.
\end{abstract}

Crown Copyright (c) 2012 Published by Elsevier B.V. All rights reserved.

\section{Introduction}

Silicon is the second most abundant material in the earth's crust and constitutes $90 \%$ of total revenue of all semiconductor industries combined together. Additionally, it is an ideal optical material for weight sensitive infrared (IR) applications and other high added value products such as X-ray optics and X-ray interferometers. Single point diamond turning (SPDT) is one established way of obtaining mirror finished surfaces on a variety of brittle materials [1-5]. SPDT offers the highest efficiency for generating complex shapes with good quality optical surfaces right up to the edge of the element [6] and hence is preferred over other ultra precision manufacturing processes such as grinding and polishing. SPDT was established for manufacturing of silicon during the late 1980s exploiting a so-called "brittle-ductile transition (BDT)" phenomenon [7-9]. However, ductile regime machining of large size silicon components by SPDT becomes a challenging task due

\footnotetext{
* Corresponding author. Tel.: +44 01314513197; fax: +44 01314513129

E-mail address: x.luo@hw.ac.uk (X. Luo).
}

to rapid degradation of the diamond tool by the wear causing a sudden transition of the material removal mechanism from ductile mode to brittle fracture in the cutting region with consequent deterioration to the quality of cut.

A lot of experimental work has been done so far to characterize the tool wear [10-13]. However, the time scales over which these experiments have been conducted are too long to permit any direct observation of the phenomena occurring at the atomic level which normally take place at the femtosecond to picosecond timescale. This is the reason that experimental studies cannot reveal much more than what is already known and hence were unsuccessful addressing the root cause of tool wear. Therefore, molecular dynamics (MD) simulations have been employed to simulate nanometric turning processes since the 1980s $[14,15]$. However, most of the simulations performed to date have assumed either a rigid tool or have utilized a Morse potential energy function to demonstrate the influence of cutting variables on the tool wear [16-19] including the one reporting the formation of "dynamic hard particles" [20].

Earlier studies have not taken account of the fact that the Morse potential function is di-atomic pair-potential which involves the interaction of only two atoms to obtain the resulting force and 
hence is not robust enough to describe covalent bond interactions between silicon and diamond. Hence, a more realistic potential energy function may produce better insights into SPDT of silicon. Therefore, this paper adopts an ABOP formalism based three-body potential energy function proposed by Erhart and Albe [21] to describe the interactions within and between the diamond tool and single crystal silicon workpiece to understand the influence of temperature and crystal orientation on tool wear. Using the simulation results, a mechanism has been proposed which is in excellent agreement with a recently reported experimental study.

\section{MD simulation}

A public-domain computer code, known as "Large-scale atomic/molecular massively parallel simulator" (LAMMPS) [22] was used to perform the MD simulation while Visual Molecular Dynamics (VMD) [23] and OVITO [24] were used for the enhanced visualization of atomistic data. The following paragraphs give details of the implementation of this code for the simulation in hand.

\subsection{MD simulation model}

The essence of nanometric cutting simulation through MD is simply a classical solver of Newton's second law of motion, where the atoms in the Newton and thermostatic zones are assumed to follow Newton's second law as follows:

$a_{i x}=\frac{F_{i x}}{m_{i}}=\frac{d^{2} x_{i}}{d t^{2}}, \quad F_{i x}=-\frac{d V}{d x_{i}}$

where $a_{i x}$ represents the $i$ th atom's acceleration in the $x$ direction and $m_{i}$ is the mass of the $i$ th atom. $F_{i x}$ is the interaction force acting on the $i$ th atom by the $j$ th atom in the $x$ direction, $x_{i}$ is ith atom's $x$-coordinate and $V$ is the potential energy function. A schematic diagram of the nanometric cutting simulation model is shown in Fig. 1. The model developed in this work is based on a fixed base and side which was found more appropriate to study nanometric cutting process [25]. Moreover, negative tool rake angles have been found more conducive to machine brittle materials so the model incorporates this [15].

Both the single-crystal silicon workpiece and the diamond cutting tool were modelled as deformable bodies in order to study the tribological interactions between the two. This is in contrast to previous simulations which have assumed the cutting tool to be a rigid body, a reasonable assumption if the focus of interest is the mechanism of nanometric cutting rather than the tool wear $[17,26,27]$. In the simulation model shown in Fig. 1, the cutting tool and workpiece are divided into three different zones: Newton atoms, thermostatic atoms and boundary atoms.

The boundary atoms are assumed to remain unaffected during the simulation and thus remain fixed in their initial lattice positions, serving to reduce the boundary effects and maintain the symmetry of the lattice. In conventional machining operations, the energy from plastic deformation in the primary shear zone and friction at the tool-chip interface generate heat, which is carried away by chips and lubricants, and is conducted into the tool and workpiece. The nanometric cutting model is, however, extremely small and is not capable of dissipating the cutting heat itself. The motion of the thermostatic atoms is therefore re-scaled to a temperature of $300 \mathrm{~K}$ at every time step. The velocity of the atoms can be used to compute the local temperature of the atoms using the relationship between kinetic energy and temperature:

$\frac{1}{2} \sum_{i} m_{i} v_{i}^{2}=\frac{3}{2} N k_{b} T$ where $N$ is the number of atoms, $v_{i}$ represents the velocity of the $i$ th atom, $k_{b}$ is the Boltzmann constant $\left(1.3806503 \times 10^{-23} \mathrm{~J} / \mathrm{K}\right)$ and $T$ represents the atomistic temperature. However, the instantaneous fluctuations in kinetic energy per atom would be very high so these are averaged temporally and/or spatially over few timesteps and reassigned to each atom at every $N$ steps to be converted into equivalent temperature. It should be noted here that the movement of the tool will also contribute to the kinetic energy so the component of tool displacement was accordingly subtracted and incorporated in the simulation.

Dislocations play a crucial role in the plastic deformation of materials. The thermal vibrations of atoms at finite temperatures make it difficult to observe dislocations during MD simulations. The widely used methods for tracing such dislocations and other lattice defects are coordinate number, slip vector and centro-symmetry parameter (CSP). Owing to thermal vibration of atoms, CSP has been proposed as an effective measure compared to other methods [28] A CSP can be computed using the formula:

$\mathrm{CSP}=\sum_{i=1}^{N / 2}\left|\overrightarrow{R_{i}}+\overrightarrow{R_{i+(N / 2)}}\right|^{2}$

where $N$ nearest neighbours of each atom are identified and $R_{i}$ and $R_{i+(N / 2)}$ are vectors from the central atom to a particular pair of nearest neighbours. Thus, the number of possible neighbour pairs is $N \times(N-1) / 2$. More details about CSP have been furnished elsewhere [29].

\subsection{Selection of potential energy function}

The accuracy of the potential function governs the reliability of an MD simulation. The analytical bond order potential (ABOP) formalism based potential energy function proposed by Erhart and Albe [21] can describe both dimer and bulk properties of silicon, for this reason, is claimed to be better than the bond order potential (BOP) formalism based Tersoff potential energy function [30,31]. Additionally, it is a three-body potential function which allows formation and breaking of bonds during the machining simulation which is vital in understanding tool wear. Therefore, ABOP formalism was used to describe $\mathrm{Si}-\mathrm{Si}, \mathrm{C}-\mathrm{C}$ and $\mathrm{Si}-\mathrm{C}$ interactions as follows:

Total energy $E=\sum_{i>j} f_{c}\left(r_{i j}\right)[V_{R}\left(r_{i j}\right)-\underbrace{\frac{b_{i j}+b_{j i}}{2}}_{\overline{b_{i j}}} V_{A}\left(r_{i j}\right)]$

where $E$ is the cohesive energy which is the sum of individual bond energies with following repulsive and attractive contributions:

$V_{R}(r)=\frac{D_{0}}{S-1} \exp \left[-\beta \sqrt{2 S}\left(r-r_{0}\right)\right]$

$V_{A}(r)=\frac{S D_{0}}{S-1} \exp \left[-\beta \sqrt{2 / S}\left(r-r_{0}\right)\right]$

where $D_{0}$ and $r_{0}$ are the dimer energy and bond length. The cutoff function is given by:

$f_{c}(r)= \begin{cases}1 & r<R-D \\ 0 & r>R+D \\ \frac{1}{2}-\frac{1}{2} \sin \left(\frac{\pi}{2} \frac{r-R}{D}\right) & |R-r| \leq D\end{cases}$

where parameters $R$ and $D$ specify the position and the width of the cutoff region. The bond order is given by:

$b_{i j}=\left(1+\chi_{i j}\right)^{-1 / 2}$ 


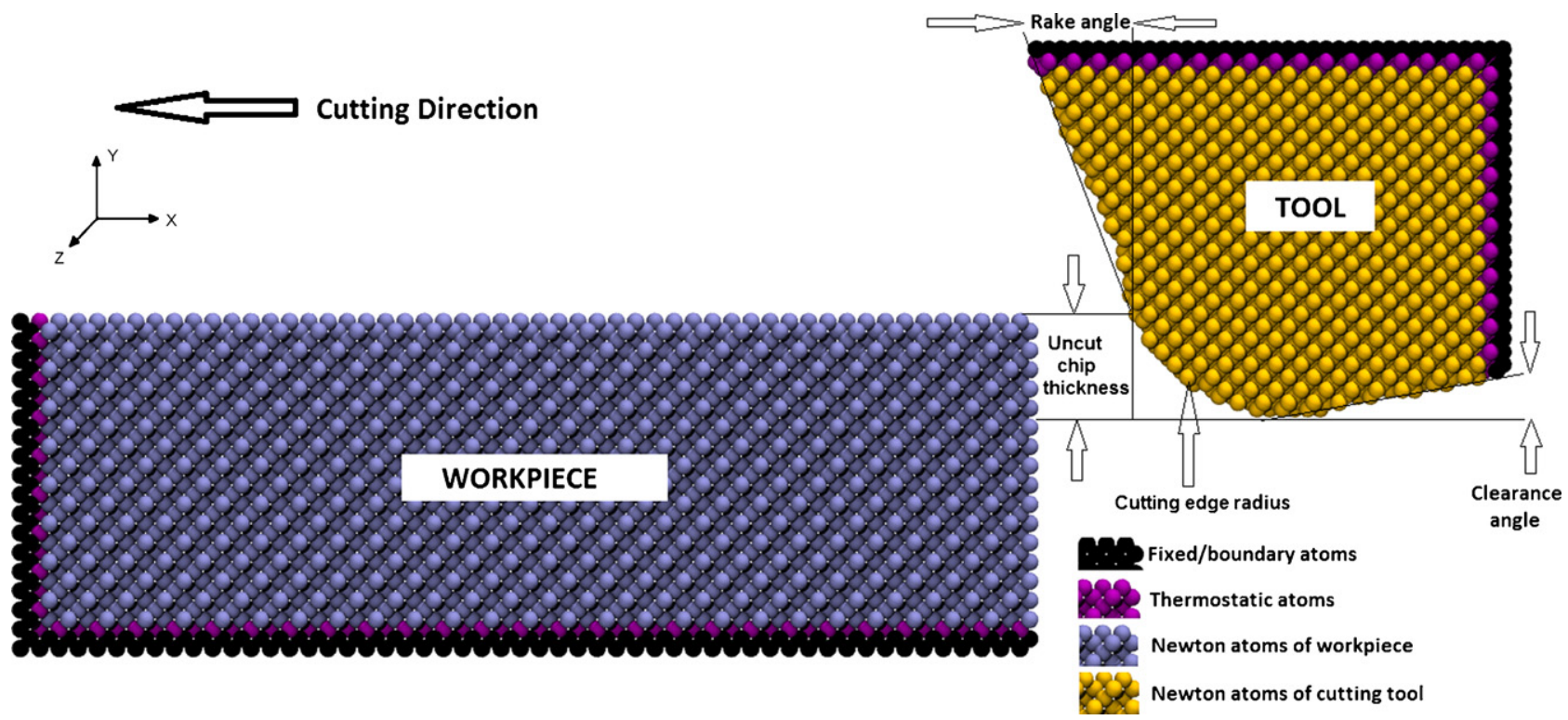

Fig. 1. Schematic of MD simulation model.

Table 1

Potential function parameters used in this study [21].

\begin{tabular}{llll}
\hline & $\mathrm{Si}-\mathrm{Si}$ & $\mathrm{C}-\mathrm{C}$ & $\mathrm{Si}-\mathrm{C}$ \\
\hline$D_{0}(\mathrm{eV})$ & 3.24 & 6 & 4.36 \\
$r_{0}(\AA)$ & 2.222 & 1.4276 & 1.79 \\
$S$ & 1.57 & 2.167 & 1.847 \\
$\beta\left(\AA^{-1}\right)$ & 1.476 & 2.0099 & 1.6991 \\
$\gamma$ & 0.09253 & 0.11233 & 0.011877 \\
$c$ & 1.13681 & 181.910 & 273987 \\
$d$ & 0.63397 & 6.28433 & 180.314 \\
$h$ & 0.335 & 0.5556 & 0.68 \\
$2 \mu\left(\AA^{-1}\right)$ & 0 & 0 & 0 \\
$R(\AA)$ & 2.9 & 2 & 2.4 \\
$D(\AA)$ & 0.15 & 0.15 & 0.2 \\
\hline
\end{tabular}

$\chi_{i j}=\sum_{k(\neq i, j)} f_{c}\left(r_{i k}\right) \exp \left[2 \mu\left(r_{i j}-r_{i k}\right) g\left(\theta_{i j k}\right)\right.$

and angular function is given by:

$g(\theta)=\lambda\left(1+\frac{c^{2}}{d_{2}}-\frac{c^{2}}{d^{2}+(h+\cos \theta)^{2}}\right)$

The potential function parameters used in the simulation are listed in Table 1.

\subsection{Equilibrium lattice parameter}

Use of inappropriate lattice parameters in an MD simulation will affect the total energy content of the system. The resulting thermal fluctuations could alter the defined uncut chip thickness and other machining parameters during the equilibration. Goel et al. $[32,33]$ have suggested using the equilibrium lattice parameters to minimize these thermal vibrations. Accordingly, the equilibrium lattice parameters as shown in Table 2 were used in the current

Table 2

Comparison of lattice parameters obtained through experiment and calculation.

\begin{tabular}{lll}
\hline Material & $\begin{array}{l}\text { Experimental known } \\
\text { lattice parameter at } \\
300 \mathrm{~K}(\AA)\end{array}$ & $\begin{array}{l}\text { Calculated equilibrium } \\
\text { lattice parameter at } \\
300 \mathrm{~K}(\AA)\end{array}$ \\
\hline Single crystal silicon & $5.429[21]$ & 5.429 \\
Diamond & $3.56683[34]$ & 3.5656 \\
\hline
\end{tabular}

simulation to obtain accurate simulation results. It can be seen that both experimental and theoretical values are in close agreement with each other. A close agreement between the equilibrium lattice parameter and experimental lattice parameter is in fact one of the criteria for the validation of the potential function itself.

\subsection{MD simulation setup}

The MD simulation model was developed by replicating the unit cell using periodic boundary conditions. However, the periodic box dimensions must be chosen carefully. Since the MD simulation model in the current case has two diamond cubic lattices with different lattice parameters i.e. $3.5656 \AA$ and $5.429 \AA$, for a $3 / 2$ ratio, it will have a mismatch of almost $1.5 \%$. Hence, an arbitrary chosen periodic box dimension may cause a strained system while generating atoms in the lattice. In order to accommodate two different lattices with periodic boundaries, the periodic box dimensions must be chosen so that the lattice parameters are in an integer relation. Alternatively, this could be compensated by increasing the size of the simulation model. This was then followed by energy minimization to avoid overlaps in the positions of the atoms. The simulation model was equilibrated to $300 \mathrm{~K}$ under the micro canonical (NVE) ensemble and the initial velocities of the atoms were assigned in accordance with a Maxwell-Boltzmann distribution. During the equilibration process, the total energy is not conserved and so the trajectories should not be used to compute any properties while the potential energy continues to convert to kinetic energy and vice versa. This procedure causes the temperature to fluctuate until it becomes stationary. Once sufficient time has been given for equilibration, the velocity scaling is removed and the system then follows NVE dynamics. In the current work, three simulation cases involving different combinations of crystal orientations were considered. The details of these crystal orientations along with other variables implemented in the simulation are listed in Table 3.

\section{Results and discussions}

It has been widely recognized that it is the high pressure phase transformation (HPPT), known the Herzfeld-Mott transition [35] that causes metallization of group-IV elements such as silicon and germanium which are brittle at room temperature [36]. 
Table 3

Variables used in the MD simulation model.

\begin{tabular}{ll}
\hline $\begin{array}{l}\text { Workpiece dimensions } \\
\text { Number of atoms in the } \\
\text { workpiece and tool }\end{array}$ & $42.0743 \mathrm{~nm} \times 4.6353 \mathrm{~nm} \times 3.5656 \mathrm{~nm}$ \\
$\begin{array}{l}\text { Cutting edge radius } \\
\text { Uncut chip thickness/in-feed } \\
\text { Crystal orientation }\end{array}$ & $1.313 \mathrm{~nm}$ \\
& $1.313 \mathrm{~nm}$ \\
& Three simulation cases were tested: \\
& (i) Cubic orientation of tool with cutting \\
& direction $\langle-110\rangle$ while workpiece was \\
& machined on $(111)$ orientation. \\
& (ii) Cubic orientation of tool with cutting \\
& direction $\langle 100\rangle$ while workpiece was \\
& machined on $(010)$ orientation. \\
& (iii) Dodecahedral orientation of tool with \\
& cutting direction $\langle-110\rangle$ while workpiece \\
& was machined on $(111)$ orientation. \\
& $-25^{\circ}$ and $10^{\circ}$ \\
Tool rake and clearance angle & $300 \mathrm{~K}$ \\
Equilibration temperature & $100 \mathrm{~m} / \mathrm{s}$ \\
Cutting velocity & $0.5 \mathrm{fs}$ \\
Timestep &
\end{tabular}

Such transformations are metallic as they cause closure of the valence-conduction band gap due to overlap of wave functions and delocalization of the valence electrons [37]. High magnitude of stress (hydrostatic + deviatoric) cause a phase change of silicon from its stable diamond cubic (alpha-silicon) to the body centred tetragonal (beta-silicon) lattice structure $[38,39]$. The magnitude of transition pressure causing such transformations in silicon has been reported to be in the range of 11-13 GPa which brings a simultaneous reduction in the atomic volume of silicon by an extent of $22 \%$ [40].

Thus, HPPT in silicon the sole reason for the BDT phenomenon observed experimentally via Raman scattering and laser microRaman spectroscopy [41,42]. In the subsequent sections, the evidence for HPPT and the consequent effects of temperature and crystal orientation on tool wear are discussed.

\subsection{High pressure phase transformation (HPPT)}

Fig. 2 represents the variation in coordination number after the tool has advanced by $1 \mathrm{~nm}$ while the workpiece is machined on an (010) surface along the $\langle 100\rangle$ direction using the cubic orientation of the tool. It can be seen from Fig. 2 that the bulk of the single crystal silicon has a coordination number of 4 which is indicative of a covalently bonded system in a diamond cubic structure. Coordination values of 1 and 2 represent termination of bonds (dangling bonds) on the surface of the silicon workpiece. During nanometric cutting, atoms with a coordination value of 4 decreased with a corresponding increase in the number of atoms with coordination values of 5 and 6 . A change in coordination value from 4 to 6 during nanometric cutting confirms the formation of the betasilicon structure as observed in the past. Hence, it is reasonable to

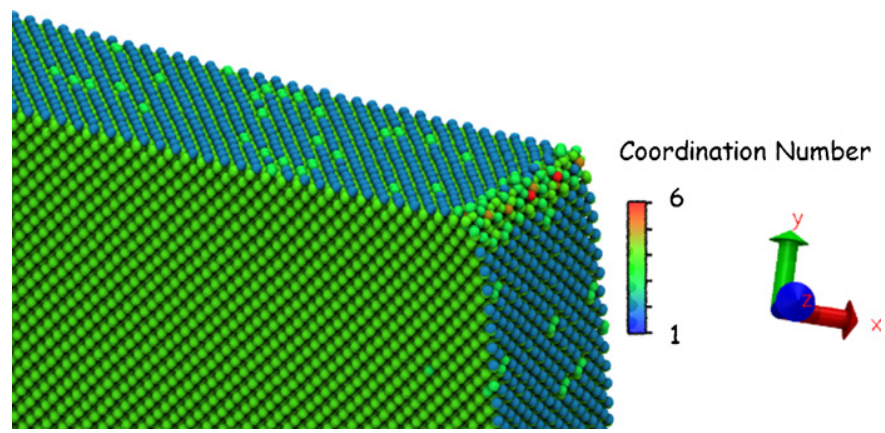

Fig. 2. Snapshot showing variation in coordination number after $1 \mathrm{~nm}$ of tool advance.

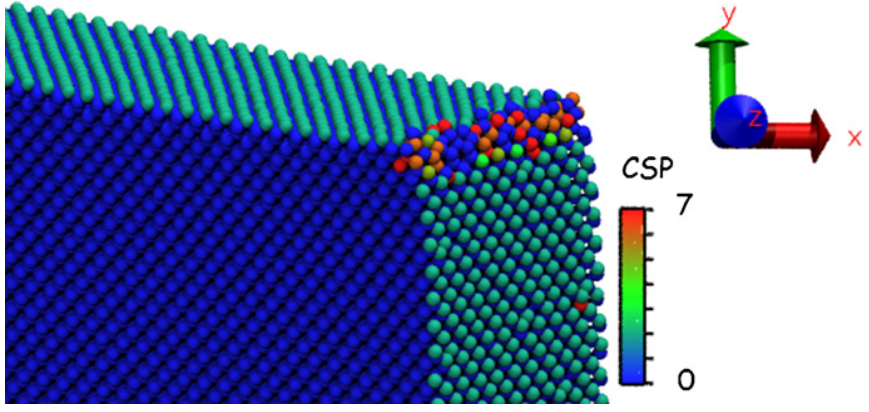

Fig. 3. Snapshot showing variation in centro symmetry parameter after $1 \mathrm{~nm}$ of tool advance.

conclude that, during nanometric cutting, there occurs a phase change of silicon from its stable diamond cubic (alpha-silicon) structure to a body centred tetragonal (beta-silicon) structure.

It is interesting to note that alongside HPPT, few atoms were also found with a coordination value of 1 or 2 which signifies some newly formed dangling bonds of silicon at the cutting zone.

Fig. 3 represents the variation in centro symmetry parameter (CSP) after the tool has advanced by $1 \mathrm{~nm}$ while the workpiece is machined on an (010) surface along an $\langle 100\rangle$ direction using the cubic orientation of the tool. The zero value of CSP in Fig. 3 corresponds to an atom surrounded by other atoms on a perfect lattice. The positive value of CSP corresponds to lattice disorder including the surface atoms. A higher positive value of CSP in the cutting zone is another confirmation of lattice disorder during the nanometric machining of silicon. Unlike, coordination number, the positive value of CSP does not suggest any phase of material and may go up to any value depending on the cutting conditions. However, a comparison of Fig. 2 and Fig. 3 clearly suggests that there is a lot more consistency in the colour of surface atoms in the CSP measure compared to coordination number. This in turn confirms that CSP is a more effective measure of the dislocations/lattice disorder in a thermally sensitive environment.

\subsection{Temperature and its effect}

Fig. 4 shows the average temperature rise during the machining of a silicon workpiece while machining on an (010) surface along $\langle 100\rangle$ direction using the cubic orientation of the diamond tool.

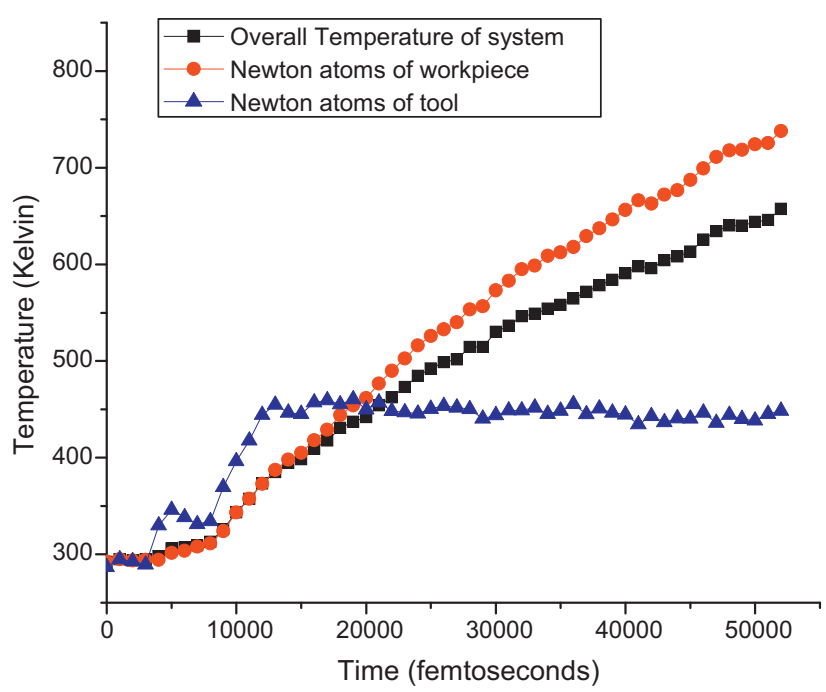

Fig. 4. Temperature rise during nanometric cutting of silicon. 


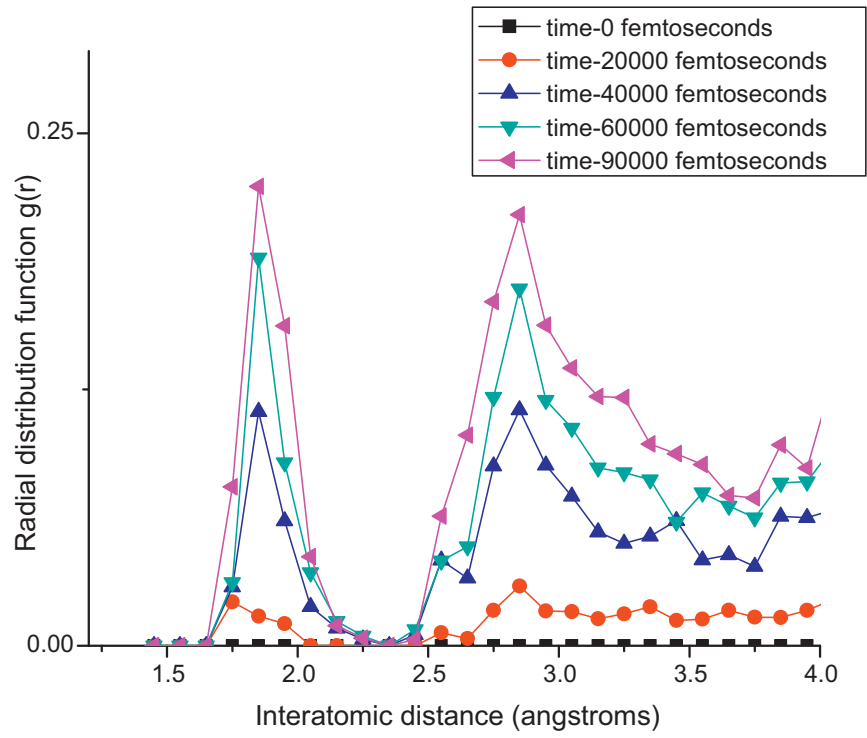

Fig. 5. Radial distribution function between atoms of diamond tool and silicon workpiece.

The maximum temperature $(\sim 750 \mathrm{~K})$ during the simulation was found to be in the primary shear zone and towards the end of the flank face of the tool, where silicon atoms recover elastically after being heavily compressed by the cutting tool. Another observation worth noting from Fig. 4 is that the average rise in the temperature of the diamond tool remains steady at a value of around $450 \mathrm{~K}$ until the end of cutting. This stability may be attributed to the high thermal conductivity of diamond which enables quick dissipation of heat. It may be recalled here that the HPPT in the machining zone was accompanied by the formation of dangling bonds of silicon. Under the prevailing conditions of high temperature in the cutting zone and presence of chemically active dangling bonds, a single phase solid state chemical reaction $[43,44]$ between the newly formed dangling bonds of silicon with the pre-existing dangling bonds of the diamond tool leads to the formation of silicon carbide as follows:

$$
\mathrm{Si}(\mathrm{s}, \mathrm{l}, \mathrm{g})+\mathrm{C} \rightarrow \mathrm{SiC}
$$

The formation of silicon carbide during the MD simulation was confirmed through the radial distribution between silicon workpiece and the diamond tool which is plotted at intervals of $10,000 \mathrm{fs}$ in Fig. 5.

In Fig. 5, at timestep 0, $g(r)$ was 0 which means there was no existing bond between silicon and carbon before the contact between the tool and the workpiece. However, with increasing time or tool advancement, it was observed that a peak continued to grow at an inter-atomic distance of $1.9 \AA$ with a second peak at around $3.08 \AA$. It is known that tetrahedral geometry of a compound of silicon and carbon i.e. silicon carbide $(\mathrm{SiC})$ possess the same bond length and interplanar spacing. Hence, this is a clear indication of the formation of silicon carbide during SPDT of silicon. The newly formed silicon carbide further accelerates the wear of the diamond tool through abrasive processes [32]. In order to suppress wear, an appropriate coolant can thus be used to regulate the temperature rise and to create a protective film which can reduce chemical contact between diamond and silicon.

The extent of tool flank wear remains higher than rake wear. This can be attributed to the temperature gradient on the tool rake and tool flank face as shown in Fig. 6. It can be seen that the number of atoms showing an increase in temperature is larger at the tool flank face than the rake face especially towards the end of the tool flank face where silicon atoms try to recover elastically. Accordingly, it is plausible to assert that high temperatures at the tool flank face will accelerate the rate of formation of silicon carbide. Since silicon carbide is known to be harder than silicon, abrasion of SiC with a diamond tool results in $s p^{3}-s p^{2}$ hybridization of diamond [32], the eventual outcome of which would be the disordered graphitization of diamond. Hence, both these cycles i.e. formation of $\mathrm{SiC}$ and graphitization of diamond tool proceed in tandem with each other during SPDT of silicon.

The wear mechanism described here seems to confirm the experimental study where Zong et al. [45] used X-ray photoelectron spectroscope (XPS) to observe silicon carbide and carbon like particles in the wafer surface layers generated after machining of single crystal silicon with a diamond tool.

\subsection{Influence of crystal orientation}

Sharif Uddin et al. [11] have recommended the use of the dodecahedral orientation of a diamond tool while Cheng et al. [46] have

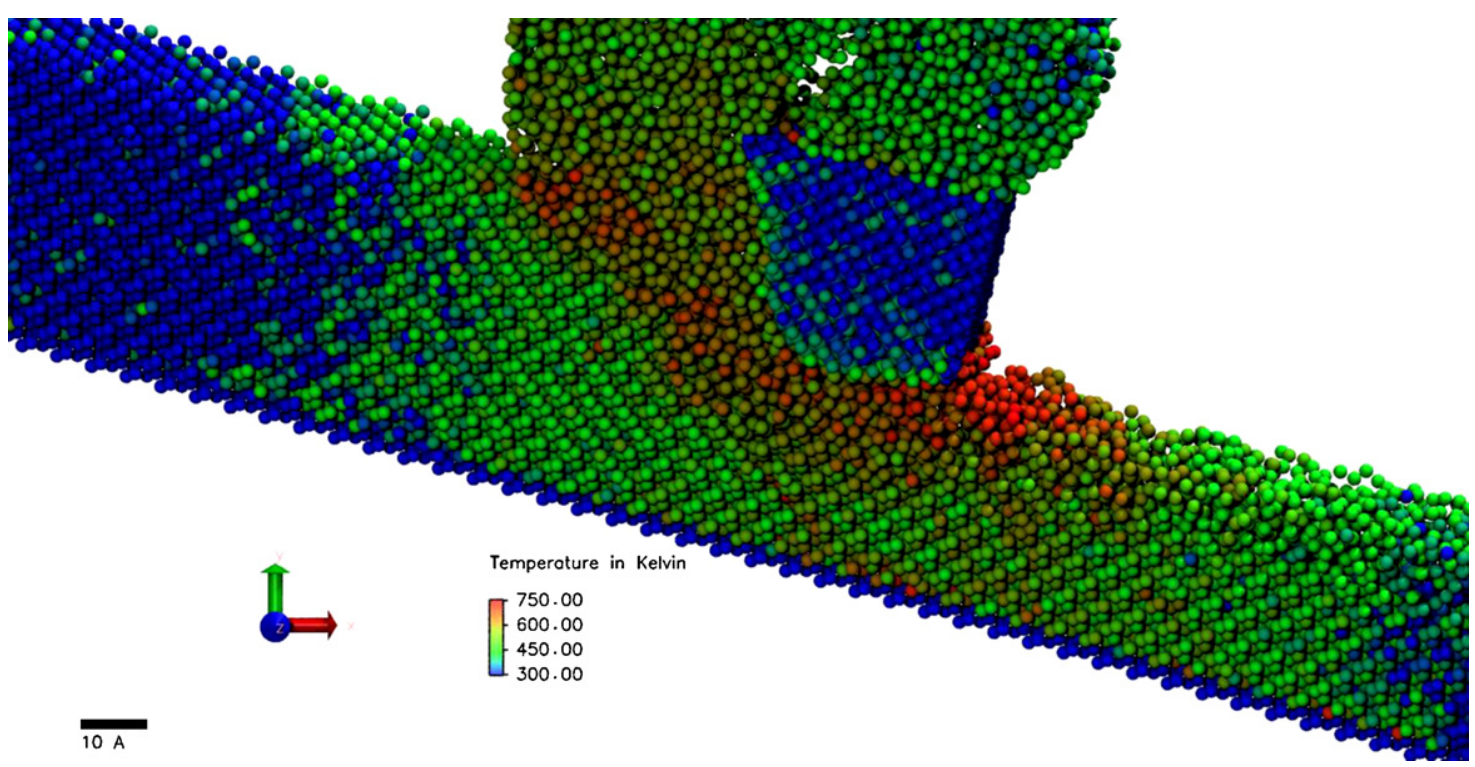

Fig. 6. Temperature distribution on atoms of diamond tool during machining. 


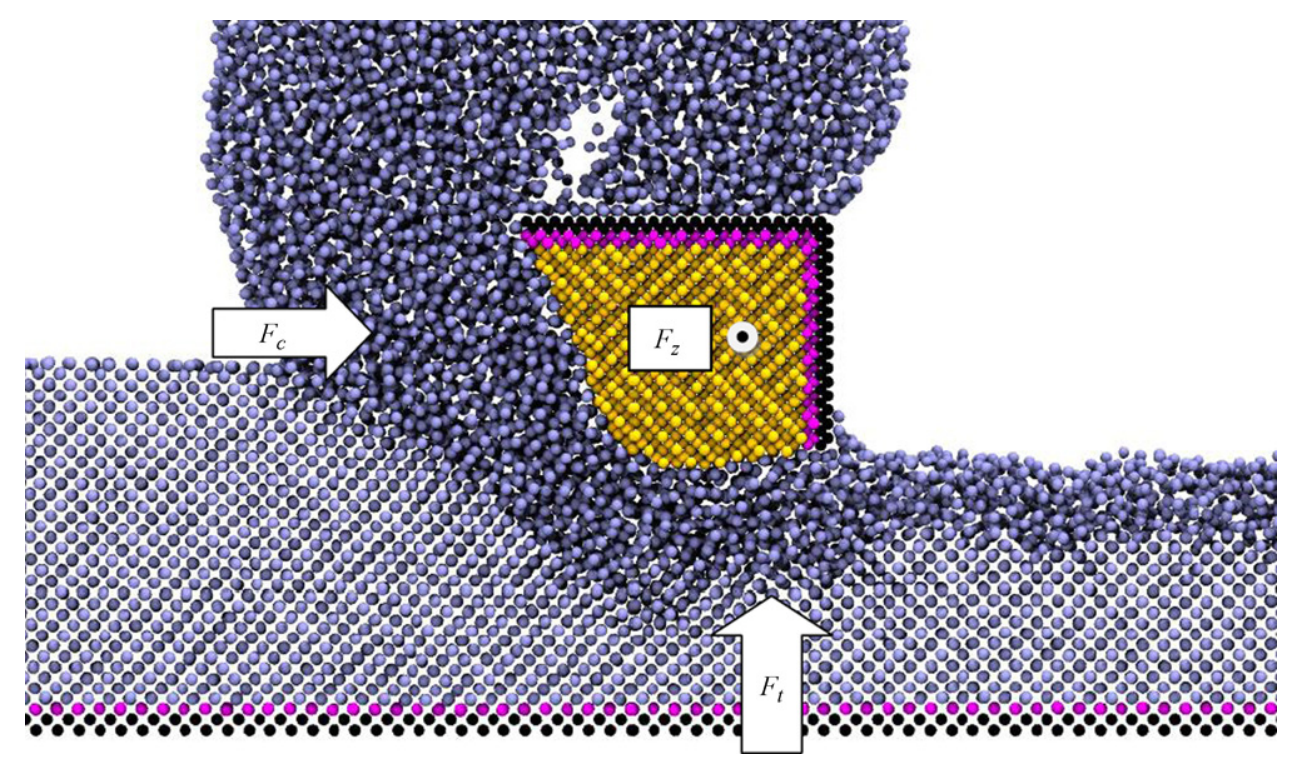

Fig. 7. Evolution of cutting forces during nanometric cutting.

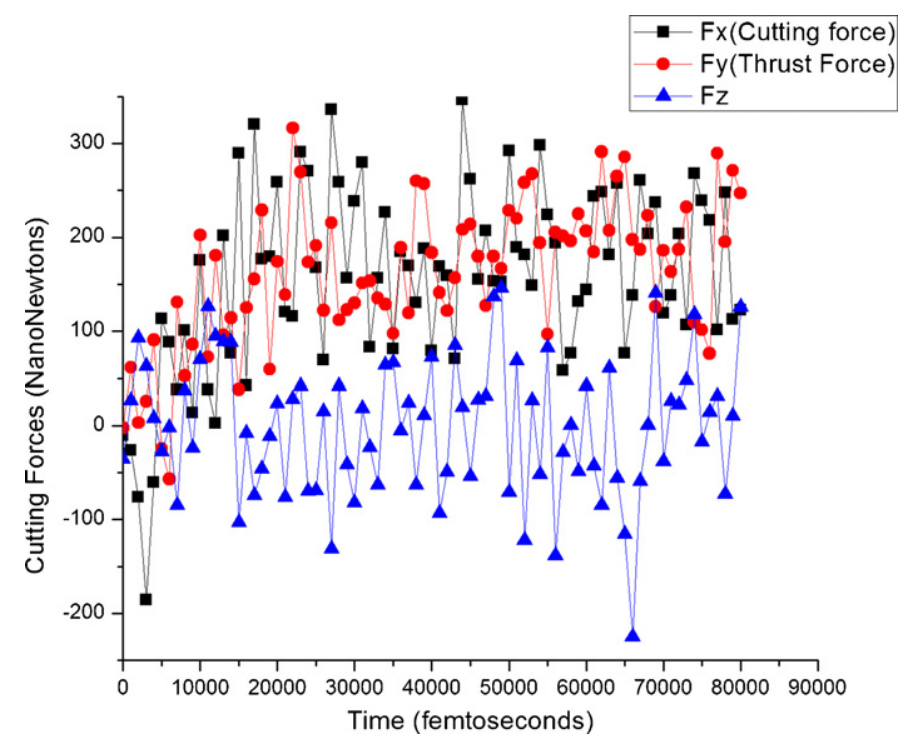

Fig. 8. Results from MD simulation showing variation in cutting forces and thrust forces. suggested using the cubic orientation in order to have better tool life. In the present work, three simulation cases were considered with different combinations of crystal orientations to investigate their effect on thrust forces.

Fig. 7 shows schematically the orientation of the components of cutting force acting on the tool. The "cutting force" $\left(F_{c}\right)$ acts in the $x$ direction, the "thrust force" $\left(F_{t}\right)$ acts in the $y$ direction and $F_{z}$ acts in the direction orthogonal to the $\mathrm{X}$ and $\mathrm{Y}$ planes.

The evolution of various forces while silicon is machined on an $(010)$ surface a $\langle 100\rangle$ direction using the cubic orientation of the tool is shown in Fig. 8.

The selection of crystal orientation becomes vital while dealing with anisotropic materials like diamond. The evolution of thrust forces with change in crystal orientation can thus be a useful criterion for identifying the appropriate crystal orientation for practical purposes. Two of the most popularly used crystal orientations for diamond as a tool material shown in Fig. 9 are the dodecahedral and cubic orientations.

Table 4 and Fig. 10 show the results obtained for the thrust forces while cutting silicon with different combinations of crystal orientations of tool and workpiece. It can be seen that the slope and amplitude of thrust forces is minimum using the (111) orientation of the workpiece with the cubic orientation of the diamond tool.

This confirms that cubic orientation of the diamond tool will provide better tool life as was suggested by Cheng et al. [46] on
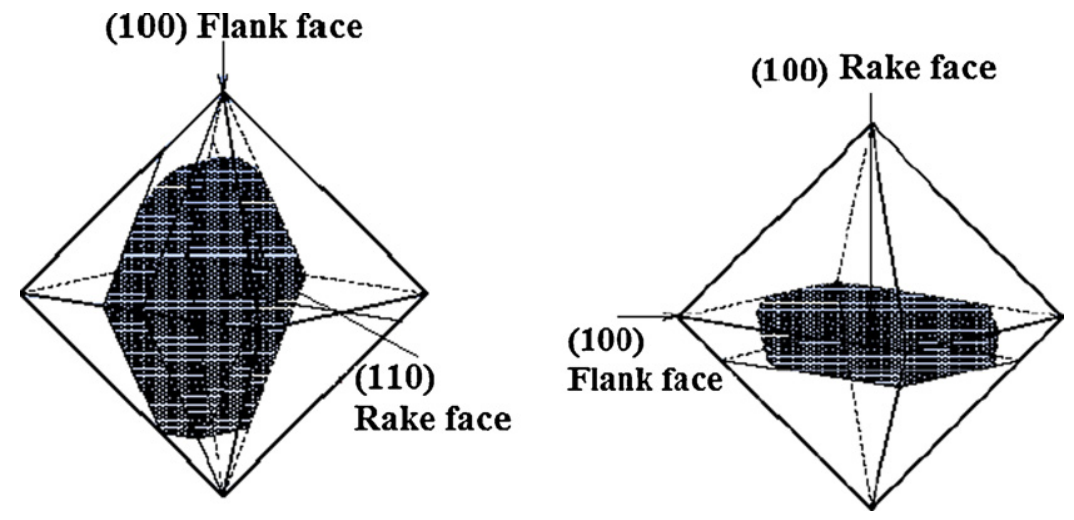

Fig. 9. Popular crystal orientation of the diamond tool. (a) Dodecahedral orientation; (b) cubic orientation. 
Table 4

Calculation of cutting forces with different crystal orientation.

\begin{tabular}{|c|c|c|c|c|}
\hline S.N. & Orientation of silicon workpiece & Cutting direction & Orientation of diamond tool & Magnitude of thrust forces observed \\
\hline 1 & $(111)$ & $\langle-110\rangle$ & Cubic & Minimum \\
\hline 2 & $(010)$ & $\langle 100\rangle$ & Cubic & Intermittent \\
\hline 3 & (111) & $\langle-110\rangle$ & Dodecahedral & Maximum \\
\hline
\end{tabular}

\section{Stacked comparison of forces with different orientations}

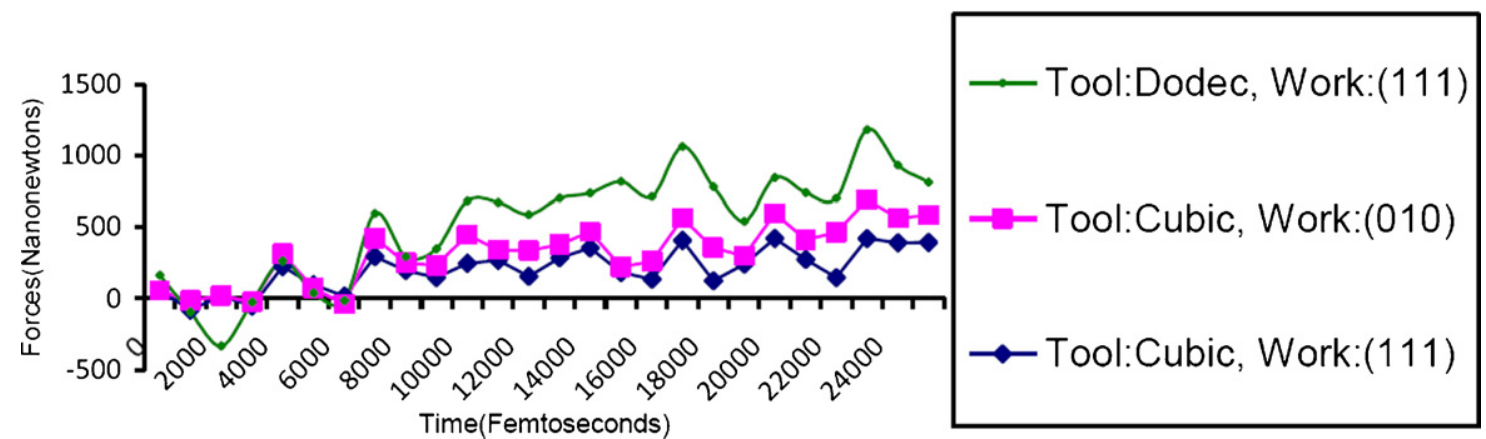

Fig. 10. Variation in thrust forces using different crystal orientations.

account of its higher micro strength and lower wear rate. Fig. 10 also consistent with the theoretical cleavage energy of the (111) plane hence confirming that machining of silicon on (111) plane will require least energy.

\section{Conclusions}

An MD simulation model has been used to develop a quantitative and qualitative understanding of the influence of crystal orientation and temperature on tool wear during single point diamond turning of silicon. The following conclusions can be drawn accordingly:

1. High pressure phase transformation of silicon to form $\mathrm{Si}-\mathrm{II}$ is a necessary condition for ductile regime machining of silicon. As long as this condition is achieved, it is possible to machine silicon in the ductile regime even with a blunt tool.

2. Formation of silicon carbide and simultaneous $s p^{3}-s p^{2}$ hybridization of the diamond tool represent the basic wear cycle of cutting tools during SPDT of silicon.

3. Temperature gradient between the tool rake and flank face cause relatively higher flank wear which may be minimized by using an appropriate coating e.g. Perfluoropolyether (PFPE) polymer on diamond tool.

4. The lower thrust forces with the cubic orientation of the diamond tool compared to the dodecahedral orientation signify that the cubic orientation is highly wear resistant and hence more appropriate for practical use.

5. The three-body potential energy function used in the simulation is far more effective for studying tool wear compared to a pair potential, such as Morse potential function.

\section{References}

[1] B. Krauskopf, Diamond turning: reflecting the demands of precision, Manufacturing Engineering 92 (1984) 90-100.

[2] T. Nakasuji, S. Kodera, S. Hara, et al., Diamond turning of brittle materials for optical components, CIRP Annals - Manufacturing Technology 39 (1) (1990) 89-92.

[3] M.A. Davies, C.J. Evans, S.R. Patterson, et al., Application of precision diamond machining to the manufacture of micro-photonics components, in: Lithographic and Micromachining Techniques for Optical Component Fabrication II, SPIE, San Diego, USA, 2003.
[4] J.A. Patten, Ductile regime nanocutting of silicon nitride, in: Proceedings ASPE 2000 Annual Meeting, 2000, pp. 106-109.

[5] J. Yan, Z. Zhang, T. Kuriyagawa, Mechanism for material removal in diamond turning of reaction-bonded silicon carbide, International Journal of Machine Tools and Manufacture 49 (5) (2009) 366-374.

[6] R.L. Rhorer, C.J. Evans, Fabrication of optics by diamond turning, in: Handbook of Optics, Mcgraw Hill, 2010.

[7] B. Lawn, R. Wilshaw, Indentation fracture: principles and applications, Journal of Materials Science 10 (6) (1975) 1049-1081.

[8] R.O. Scattergood, N. Blake, Ductile-regime machining of germanium and silicon, Journal of the American Ceramic Society 73 (4) (1990) 949-957.

[9] P. Shore, Machining of Optical Surfaces in Brittle Materials using an Ultra Precision Machine Tool, PhD Thesis, Cranfield University, 1995.

[10] R.G. Jasinevicius, J.G. Duduch, et al., Dependence of brittle-to-ductile transition on crystallographic direction in diamond turning of single-crystal silicon, in: Proceedings of the Institution of Mechanical Engineers, Part B, Journal of Engineering Manufacture (2011)

[11] M. Sharif Uddin, K.H.W. Seah, X.P. Li, M. Rahman, K. Liu, Effect of crystallographic orientation on wear of diamond tools for nano-scale ductile cutting of silicon, Wear 257 (7-8) (2004) 751-759.

[12] C.J. Wong, Fracture and wear of diamond cutting tools, Journal of Engineering Materials and Technology 103 (4) (1981) 341-345.

[13] J. Yan, K. Syoji, J. Tamaki, Some observations on the wear of diamond tools in ultra-precision cutting of single-crystal silicon, Wear 255 (7-12) (2003) 1380-1387.

[14] J.F. Belak, I.F. Stowers, A molecular dynamics model of orthogonal cutting process, in: Proceedings of American Society Precision Engineering Annual Conference, 1990, pp. 76-79.

[15] R. Komanduri, N. Chandrasekaran, L.M. Raff, Effect of tool geometry in nanometric cutting: a molecular dynamics simulation approach, Wear 219 (1) (1998) 84-97.

[16] K. Maekawa, A. Itoh, Friction and tool wear in nano-scale machining - a molecular dynamics approach, Wear 188 (1-2) (1995) 115-122.

[17] R. Komanduri, N. Chandrasekaran, L.M. Raff, Molecular dynamics simulation of the nanometric cutting of silicon, Philosophical Magazine Part B 81 (12)(2001) 1989-2019.

[18] H. Tanaka, S. Shimada, N. Ikawa, Brittle-ductile transition in monocrystalline silicon analysed by molecular dynamics simulation, in: Proceedings of the Institution of Mechanical Engineers, Part C, Journal of Mechanical Engineering Science 218 (6) (2004) 583-590.

[19] Z. Wang, L. Yingchun, M. Chen, Z. Tong, J. Chen, Analysis about Diamond Tool Wear in Nano-metric Cutting of Single Crystal Silicon using Molecular Dynamics Method, SPIE, 2010.

[20] M.B. Cai, X.P. Li, M. Rahman, Characteristics of dynamic hard particles in nanoscale ductile mode cutting of monocrystalline silicon with diamond tools in relation to tool groove wear, Wear 263 (7-12) (2007) 1459-1466.

[21] P. Erhart, K. Albe, Analytical potential for atomistic simulations of silicon, carbon, and silicon carbide, Physical Review B 71 (3) (2005) 035211.

[22] S. Plimpton, Fast parallel algorithms for short-range molecular dynamics, Journal of Computational Physics 117 (1995) 1-19.

[23] W. Humphrey, A. Dalke, K. Schulten, VMD - visual molecular dynamics, Journal of Molecular Graphics 14 (1996) 33-38. 
[24] A. Stukowski, Visualization and analysis of atomistic simulation data with OVITO - the Open Visualization Tool, Modelling and Simulation in Materials Science and Engineering 18 (1) (2010).

[25] Z.G. Zhang, F.Z. Fang, X.T. Hu, et al., Molecular dynamics study on various nanometric cutting boundary conditions, Journal of Vacuum Science \& Technology B 27 (3) (2009) 1355-1360.

[26] M.B. Cai, et al., Crack initiation in relation to the tool edge radius and cutting conditions in nanoscale cutting of silicon, International Journal of Machine Tools and Manufacture 47 (3-4) (2007) 562-569.

[27] R. Promyoo, H. El-Mounayri, X. Yang, Molecular dynamics simulation of nanometric cutting, Machining Science and Technology 14 (4) (2010) 423-439.

[28] Q.X. Pei, C. Lu, H.P. Lee, Large scale molecular dynamics study of nanometric machining of copper, Computation Materials Science 41 (2) (2007) 177-185.

[29] C.L. Kelchner, S.J. Plimpton, J.C. Hamilton, Dislocation nucleation and defect structure during surface indentation, Physical Review B 58 (17) (1998) 11085.

[30] J.Tersoff, Modeling solid-state chemistry: interatomic potentials for multicomponent systems, Physical Review B 39 (8) (1989) 5566.

[31] J. Tersoff, Erratum: modeling solid-state chemistry: interatomic potentials for multicomponent systems, Physical Review B 41 (5) (1990) 3248.

[32] S. Goel, X. Luo, R.L. Reuben, Molecular dynamics simulation model for the quantitative assessment of tool wear during single point diamond turning of cubic silicon carbide, Computation Materials Science 51 (1) (2012) 402-408.

[33] S. Goel, X. Luo, R.L. Reuben, et al., Replacing diamond cutting tools with CBN for efficient nanometric cutting of silicon, Materials Letters 68 (0) (2012) 507-509.

[34] http://www.siliconfareast.com/lattice_constants.htm, Lattice Constants (accessed 09.04.11).

[35] J.J. Gilman, Insulator-metal transitions at microindentations, Journal of Materials Research 7 (1992) 535-538.
[36] V. Domnich, Y. Gogotsi, Phase transformations in silicon under contact loading Reviews on Advanced Materials Science 3 (1) (2001).

[37] Y.G. Gogotsi, A. Kailer, K.G. Nickel, Pressure-induced phase transformations in diamond, Journal of Applied Physics 84 (3) (1998) 1299-1304.

[38] M.B. Cai, X.P. Li, M. Rahman, High-pressure phase transformation as the mechanism of ductile chip formation in nanoscale cutting of silicon wafer, in: Proceedings of the Institution of Mechanical Engineers, Part B, Journal of Engineering Manufacture 221 (10) (2007) 1511-1519.

[39] R. Komanduri, L. Raff, A review on the molecular dynamics simulation of machining at the atomic scale, in: Proceedings of the Institution of Mechanical Engineers, Part B, Journal of Engineering Manufacture 215 (12) (2001) 1639-1672.

[40] H.A. Abdel-Al, S.T. Smith, Thermal modeling of silicon machining - issues and challenges, in: ASPE Spring Topical Meeting: Silicon Machining, Carmel-bythe-Sea, California, 1998

[41] P.S. Pizani, R. Jasinevicius, J.G. Duduch, et al., Ductile and brittle modes in single-point-diamond-turning of silicon probed by Raman scattering, Journa of Materials Science Letters 18 (14) (1999) 1185-1187.

[42] J. Yan, Laser micro-Raman spectroscopy of single-point diamond machined silicon substrates, Journal of Applied Physics 95 (4) (2004) 2094-2101.

[43] J.G. Lee, I.B. Cutler, Formation of silicon carbide from rice hulls, Ceramic Bulletin 54 (2) (1975) 195-198

[44] V.D. Krstic, Production of fine: high-purity beta silicon carbide powders, Journal of the American Ceramic Society 75 (1) (1992) 170-174.

[45] W.J. Zong, T. Sun, D. Li, et al., XPS analysis of the groove wearing marks on flank face of diamond tool in nanometric cutting of silicon wafer, International Journal of Machine Tools and Manufacture 48 (15) (2008) 1678-1687.

[46] K. Cheng, X. Luo, et al., Modeling and simulation of the tool wear in nanometric cutting, Wear 255 (2003) 1427-1432. 